\title{
Medical Diagnosis Based on Single-Valued Neutrosophic Probabilistic Rough Multisets over Two Universes
}

\author{
Chao Zhang ${ }^{1}$, Deyu Li ${ }^{1, *}$, Said Broumi ${ }^{2}$ and Arun Kumar Sangaiah ${ }^{3}$ (i) \\ 1 Key Laboratory of Computational Intelligence and Chinese Information Processing of Ministry of Education, \\ Research Institute of Big Data Science and Industry, School of Computer and Information Technology, \\ Shanxi University, Taiyuan 030006, China; zhch3276152@163.com \\ 2 Laboratory of Information Processing, Faculty of Science Ben M'Sik, University Hassan II, B.P 7955, \\ Sidi Othman, Casablanca 20000, Morocco; broumisaid78@gmail.com \\ 3 School of Computing Science and Engineering, VIT University, Vellore 632014, India; \\ arunkumarsangaiah@gmail.com \\ * Correspondence: lidysxu@163.com; Tel.: +86-351-701-8775
}

Received: 17 May 2018; Accepted: 11 June 2018; Published: 12 June 2018

\begin{abstract}
In real-world diagnostic procedures, due to the limitation of human cognitive competence, a medical expert may not conveniently use some crisp numbers to express the diagnostic information, and plenty of research has indicated that generalized fuzzy numbers play a significant role in describing complex diagnostic information. To deal with medical diagnosis problems based on generalized fuzzy sets (FSs), the notion of single-valued neutrosophic multisets (SVNMs) is firstly used to express the diagnostic information in this article. Then the model of probabilistic rough sets (PRSs) over two universes is applied to analyze SVNMs, and the concepts of single-valued neutrosophic rough multisets (SVNRMs) over two universes and probabilistic rough single-valued neutrosophic multisets (PRSVNMs) over two universes are introduced. Based on SVNRMs over two universes and PRSVNMs over two universes, single-valued neutrosophic probabilistic rough multisets (SVNPRMs) over two universes are further established. Next, a three-way decisions model by virtue of SVNPRMs over two universes in the context of medical diagnosis is constructed. Finally, a practical case study along with a comparative study are carried out to reveal the accuracy and reliability of the constructed three-way decisions model.
\end{abstract}

Keywords: single-valued neutrosophic multisets; medical diagnosis; probabilistic rough sets over two universes; three-way decisions

\section{Introduction}

In medical science and technology, it is acknowledged that disease diagnosis is a rather complicated activity for medical experts who are faced with tasks in handling varieties of uncertain diagnostic information. In order to seek the accurate diagnosis for the considered patients, it is essential for medical experts to take into account a number of related symptoms simultaneously, and this procedure might take a long time to reach a final diagnostic outcome. Considering it is meaningful to cope with the above complex decision making situation within the background of medical diagnosis, plenty of practitioners are likely to focus on the relationship between the diagnosis set and the symptom set, and varieties of achievements have been made on the basis of fuzzy approaches [1-3]. According to the FS theory established by Zadeh [4], fuzzy approaches have been extensively used in lots of medical diagnosis situations. However, the modeling tools of classical FSs are confined when multiple kinds of 
uncertainties emerge at the same time. Thus, several new notions of generalized FSs were put forward one after another during the past decades [5].

Among various generalized FSs, in view of intuitionistic fuzzy sets (IFSs) [6] lack a reasonable scheme to effectively process inconsistent and indeterminate information embedded in realistic scenarios, Smarandache [7] initiated the framework of neutrosophic sets (NSs) and neutrosophic logic, which could be seen as a generalized form of FSs, IFSs, fuzzy logic and intuitionistic fuzzy logic [8-10]. Compared with IFSs, through adding an indeterminacy membership function which is focused on separately, NSs are able to express incomplete, inconsistent and indeterminate information efficiently. Further, in order to utilize the idea of NSs to solve a broader range of practical issues, Wang et al. [11] presented a novel branch of NSs called single-valued neutrosophic sets (SVNSs), whose values of three membership functions belong to $[0,1]$. Ever since the establishment of SVNSs, many enlightening research results have been made in many real-world areas [12-21]. Recently, inspired by one typical solution to obtain an accurate diagnosis for a patient is to arrange the medical examination at different parts on a day to day basis (e.g., morning, noon and evening), Ye [22] introduced the notion of SVNMs by taking advantage of fuzzy multisets (FMs) [23] and $n$-valued refined neutrosophic logic [24]. With the support of SVNMs, the SVN membership values occur one or multiple times, which is favourable to the expression of the above SVN diagnostic information at different time intervals, hence SVNMs could process the uncertain information well, and offer medical experts a rather powerful tool to record a complicated medical diagnosis knowledge base. Until now, the studies of SVNMs are mainly concentrated on algebraic properties, similarity measures, neutrosophic multiple relations, cosine measures, and so on [25-28].

In general, medical experts are often confronted with the following two challenges in practical medical diagnosis, one is to make an accurate diagnostic conclusion for the considered patients, another one is to provide a reasonable explanation on how to obtain the result under uncertain scenarios [29-31]. Through designing possible and deterministic decision rules, rough set theory has illustrated its powerful performances for solving various decision making situations in the above challenges [32-42]. In addition, among varieties of specific rough set models, it is worth noticing that lots of them are often too strict and might require additional information when constructing approximations originated from the classical rough set theory. In order to effectively handle the above issue, by combining the rough sets with probability theory, the concept of PRSs is initiated by Wong and Ziarko [43] to let rough set models possess the error tolerance capability when processing the noisy data. Further, PRSs are developed by virtue of more powerful soft computing tools such as Bayesian decision theory, graded set inclusions, Bayesian confirmation measures, etc [44-48]. Compared with other types of rough sets, through introducing the probability theory to estimate the rough membership, PRSs permit the existence of the error tolerance by means of the introduction of thresholds. In recent years, motivated by the notion of three-way decisions $[49,50]$, several decision making methods by means of PRSs-based three-way decisions have been put forward to promote the solving efficiency of real-world problems [51-54].

In this paper, inspired by the idea of PRSs-based three-way decisions, we systematically study various probabilistic rough approximations in the background of SVNMs information by integrating SVNMs with PRSs over two universes, and propose the model of SVNPRMs over two universes. Then, we aim to investigate a three-way decisions method by utilizing the proposed SVNPRMs over two universes under the context of medical diagnosis. In light of the above discussion, we arrange the structure of the article below. In Section 2, we revisit several fundamental notions about SVNMs and PRSs. In the next section, we present the notion of SVNRMs over two universes and PRSVNMs over two universes at first, then present the notion of SVNPRMs over two universes. Section 4 constructs a medical diagnosis algorithm based on SVNPRMs over two universes, then a case study and its corresponding comparative study are carried out to show the validity of the constructed medical diagnosis algorithm. The last section concludes the contributions of the work. 


\section{Preliminaries}

In the following, we revisit several fundamental concepts which will be utilized in the latter part of the paper.

\subsection{SVNMs}

As a generalization of many concepts such as FSs, IFSs, SVNSs, etc., the definition of SVNMs and their related operations are presented below.

Definition 1. [22] Suppose that $U$ is a finite and nonempty set, a SVNM A is featured by count truth-membership of $C T_{A}$, count indeterminacy-membership of $C I_{A}$, count falsity-membership of $C F_{A}$, where $C T_{A}, C I_{A}, C F_{A}: U \rightarrow R$ for all $x \in U$. Then a $S V N M A$ is given by

$$
\left.A=\left\{\begin{array}{c}
x,\left(T_{A}^{1}(x), T_{A}^{2}(x), \ldots, T_{A}^{q}(x)\right), \\
\left(I_{A}^{1}(x), I_{A}^{2}(x), \ldots, I_{A}^{q}(x)\right), \\
\left.F_{A}^{1}(x), F_{A}^{2}(x), \ldots, F_{A}^{q}(x)\right)
\end{array}\right\rangle \mid x \in U\right\},
$$

where the truth-membership sequence, the indeterminacy-membership sequence and the falsity-membership sequence $\left(T_{A}^{1}(x), T_{A}^{2}(x), \ldots, T_{A}^{q}(x)\right), \quad\left(I_{A}^{1}(x), I_{A}^{2}(x), \ldots, I_{A}^{q}(x)\right)$ and $\left(F_{A}^{1}(x), F_{A}^{2}(x), \ldots, F_{A}^{q}(x)\right)$ are arranged in an increasing or decreasing order. Additionally, for each $i=1,2, \ldots, q$, the sum of $T_{A}^{i}(x), I_{A}^{i}(x), F_{A}^{i}(x) \in[0,1]$ fulfills the requirement $0 \leq T_{A}^{i}(x)+I_{A}^{i}(x)+F_{A}^{i}(x) \leq 3$. For the sake of convenience, a simplified form of SVNM could be expressed as $A=\left\{\left\langle x, T_{A}^{i}(x), I_{A}^{i}(x), F_{A}^{i}(x)\right\rangle \mid x \in U, i=1,2, \ldots, q\right\}$. Furthermore, we represent the set of all SVNMs on $U$ as $S V N M(U)$. For all $x \in U, A$ is called a full $S V N M$ if and only if $x=\langle 1,0,0\rangle$, while $A$ is called an empty SVNM if and only if $x=\langle 0,1,1\rangle$.

Definition 2. [22] The length of $x$ in a SVNM $A$ is represented by $L(x: A)$, where $L(x: A)=\left|C T_{A}(x)\right|=$ $\left|C I_{A}(x)\right|=\left|C F_{A}(x)\right|\left(\left|C T_{A}(x)\right|,\left|C I_{A}(x)\right|\right.$ and $\left|C F_{A}(x)\right|$ represent the cardinality of $C T_{A}(x), C I_{A}(x)$ and $C F_{A}(x)$ ). In addition, for any $A, B \in \operatorname{SVNM}(U), L(x: A, B)=\max \{L(x: A), L(x: B)\}$.

For any two SVNMs $A$ and $B$, it is noted that $L(x: A)$ might be different from $L(x: B)$ in many situations. Through adding the maximum number for the indeterminacy-membership value and the falsity-membership value, and further adding the minimum number for the truth-membership value, we could make $L(x: A)=L(x: B)$.

Definition 3. [22] For any two SVNMs $A$ and B in the universe $U$, we have

1. $A \oplus B=\left\{\left\langle x, T_{A}^{i}(x)+T_{B}^{i}(x)-T_{A}^{i}(x) T_{B}^{i}(x), I_{A}^{i}(x) I_{B}^{i}(x), F_{A}^{i}(x) F_{B}^{i}(x)\right\rangle \mid x \in U, i=1,2, \ldots, q\right\}$;

2. $A \otimes B=\left\{\left\langle x, T_{A}^{i}(x) T_{B}^{i}(x), I_{A}^{i}(x)+I_{B}^{i}(x)-I_{A}^{i}(x) I_{B}^{i}(x), F_{A}^{i}(x)+F_{B}^{i}(x)-F_{A}^{i}(x) F_{B}^{i}(x)\right\rangle \mid x \in U\right.$, $i=1,2, \ldots, q\}$;

3. the complement of $A$ is represented by $A^{c}$ such that $\forall x \in U$, $A^{c}=\left\{\left\langle x, F_{A}^{i}(x), 1-I_{A}^{i}(x), T_{A}^{i}(x)\right\rangle \mid x \in U, i=1,2, \ldots, q\right\} ;$

4. the union of $A$ and $B$ is represented by $A \cup B$ such that $\forall x \in U$, $A \cup B=\left\{\left\langle x, T_{A}^{i}(x) \vee T_{B}^{i}(x), I_{A}^{i}(x) \wedge I_{B}^{i}(x), F_{A}^{i}(x) \wedge F_{B}^{i}(x)\right\rangle \mid x \in U, i=1,2, \ldots, q\right\} ;$

5. the intersection of $A$ and $B$ is represented by $A \cap B$ such that $\forall x \in U$, $A \cap B=\left\{\left\langle x, T_{A}^{i}(x) \wedge T_{B}^{i}(x), I_{A}^{i}(x) \vee I_{B}^{i}(x), F_{A}^{i}(x) \vee F_{B}^{i}(x)\right\rangle \mid x \in U, i=1,2, \ldots, q\right\}$.

\section{2. $P R S S$}

In view of classical rough sets being rather rigorous when constructing lower/upper approximations and often requiring some additional information, Wong and Ziarko [43] took 
advantage of the probabilistic measure theory, and presented a probabilistic model of rough sets to update the method for obtaining a related rough set region.

Definition 4. [43] Suppose that $U$ is the universe of discourse, and $P$ is the probabilistic measure based on the $\sigma$ algebra. Then $(U, R, P)$ is named a probabilistic approximation space. For any $0 \leq \beta \leq \alpha \leq 1, X \subseteq U$, the lower and upper approximations of $X$ are given by

$$
\begin{aligned}
& \underline{P}_{\alpha}(X)=\{P(X \mid[x]) \geq \alpha \mid x \in U\}, \\
& \bar{P}_{\beta}(X)=\{P(X \mid[x])>\beta \mid x \in U\},
\end{aligned}
$$

where the pair $\left(\underline{P}_{\alpha}(X), \bar{P}_{\beta}(X)\right)$ is named a PRS. Moreover, by virtue of the above approximations, the positive region, negative region and boundary region of $X$ are further given by

$$
\begin{aligned}
& P O S(X, \alpha, \beta)=\underline{P}_{\alpha}(X)=\{P(X \mid[x]) \geq \alpha \mid x \in U\}, \\
& \operatorname{NEG}(X, \alpha, \beta)=U-\bar{P}_{\beta}(X)=\{P(X \mid[x]) \leq \beta \mid x \in U\}, \\
& \operatorname{BND}(X, \alpha, \beta)=\bar{P}_{\beta}(X)-\underline{P}_{\alpha}(X)=\{\beta<P(X \mid[x])<\alpha \mid x \in U\},
\end{aligned}
$$

it is noted that the above approximations reduce to classical rough sets when $\alpha=1$ and $\beta=0$, thus classical rough sets act as a special case of PRSs.

\section{Probabilistic Rough Approximations of a SVNM under the Background of Two Universes}

In this section, we aim to put forward the probabilistic rough approximations of a SVNM under the background of two universes, and eventually develop the model of SVNPRMs over two universes. To facilitate our discussion, based on the proposed relation on SVNMs from the universe $U$ to the universe $V$ and some operations, we first discuss the general rough approximations of a SVNM under the background of two universes and present the model of SVNRMs over two universes. Then, we investigate rough single-valued neutrosophic multisets on a probabilistic approximation space over two universes and propose the model of PRSVNMs over two universes. Lastly, the definition of SVNPRMs over two universes is put forward and several significant propositions of the presented model are explored.

\subsection{Relations on SVNMs Based on Two Universes and Some Operations}

In what follows, we introduce the arbitrary relation on SVNMs based on two related universes.

Definition 5. Suppose that $U, V$ are two universes of discourse, and $R$ is a relation on $S V N M$ s. Then $R$ is given by

$$
R=\left\{\left\langle(x, y), T_{R}^{i}(x, y), I_{R}^{i}(x, y), F_{R}^{i}(x, y)\right\rangle \mid(x, y) \in U \times V, i=1,2, \ldots, q\right\},
$$

Moreover, we denote the family of all relations on $S V N M$ s from $U$ to $V$ as $S V N M(U \times V)$.

In order to facilitate the latter discussions of the paper, we present subtraction and division operations, and the corresponding score functions for SVNMs as follows.

Definition 6. For any two SVNMs $A$ and $B$ in the universe $U$, we have

1. $A \ominus B=\left\{\left\langle x, \frac{T_{A}^{i}(x)-T_{B}^{i}(x)}{1-T_{B}^{i}(x)}, \frac{I_{A}^{i}(x)}{I_{B}^{i}(x)}, \frac{F_{A}^{i}(x)}{F_{B}^{i}(x)}\right\rangle \mid x \in U, i=1,2, \ldots, q\right\}$, which is valid under the requirements $A \geq B, T_{B}^{i}(x) \neq 1, I_{B}^{i}(x) \neq 0$ and $F_{B}^{i}(x) \neq 0$; 
2. $A \oslash B=\left\{\left\langle x, \frac{T_{A}^{i}(x)}{T_{B}^{i}(x)}, \frac{I_{A}^{i}(x)-I_{B}^{i}(x)}{1-I_{B}^{i}(x)}, \frac{F_{A}^{i}(x)-F_{B}^{i}(x)}{1-F_{B}^{i}(x)}\right\rangle \mid x \in U, i=1,2, \ldots, q\right\}$, which is valid under the requirements $A \leq B, T_{B}^{i}(x) \neq 0, I_{B}^{i}(x) \neq 1$ and $F_{B}^{i}(x) \neq 1$.

In the following, the corresponding score function is proposed to rank different elements in SVNMs.

Definition 7. Suppose that $x=\left\langle T_{A}^{i}(x), I_{A}^{i}(x), F_{A}^{i}(x)\right\rangle$ is an element in a SVNM, the corresponding score function of $x$ is defined below.

$$
s(x)=\left[\sum_{i=1}^{q} T_{A}^{i}(x)+\sum_{i=1}^{q}\left(1-I_{A}^{i}(x)\right)+\sum_{i=1}^{q}\left(1-F_{A}^{i}(x)\right)\right] / 3 q .
$$

For two elements in a SVNM $x_{1}$ and $x_{2}$, we have

1. If $s\left(x_{1}\right)<s\left(x_{2}\right)$, then $x_{1}<x_{2}$;

2. If $s\left(x_{1}\right)=s\left(x_{2}\right)$, then $x_{1}=x_{2}$;

3. If $s\left(x_{1}\right)>s\left(x_{2}\right)$, then $x_{1}>x_{2}$.

\subsection{SVNRMs over Two Universes}

By virtue of the above presented relations on SVNMs based on two universes, the definition of SVNRMs over two universes is put forward below.

Definition 8. Suppose that $U, V$ are two universes of discourse, and $R \in S V N M(U \times V)$ is a relation on SVNMs. Then $(U, V, R)$ is named a general approximation space over two universes based on SVNMs. For any $A \in S V N M(V), x \in U, y \in V$, the lower and upper approximations of $A$ with respect to $(U, V, R)$ are given by

$$
\begin{aligned}
& \underline{R}(A)=\left\{\left\langle x, T_{\underline{R}(A)}^{i}(x), I_{\underline{R}(A)}^{i}(x), F_{\underline{R}(A)}^{i}(x)\right\rangle \mid x \in U, i=1,2, \ldots, q\right\}, \\
& \bar{R}(A)=\left\{\left\langle x, T_{\bar{R}(A)}^{i}(x), I_{\bar{R}(A)}^{i}(x), F_{\bar{R}(A)}^{i}(x)\right\rangle \mid x \in U, i=1,2, \ldots, q\right\},
\end{aligned}
$$

where $T_{\underline{R}(A)}^{i}(x)=\wedge_{y \in V}\left[F_{R}^{i}(x, y) \vee T_{A}^{i}(y)\right], I_{\underline{R}(A)}^{i}(x)=\vee_{y \in V}\left[\left(1-I_{R}^{i}(x, y)\right) \wedge I_{A}^{i}(y)\right], F_{\underline{R}(A)}^{i}(x)=$ $\vee_{y \in V}\left[T_{R}^{i}(x, y) \wedge F_{A}^{i}(y)\right], T_{\bar{R}(A)}^{i}(x)=\vee_{y \in V}\left[T_{R}^{i}(x, y) \wedge T_{A}^{i}(y)\right], I_{\bar{R}(A)}^{i}(x)=\wedge_{y \in V}\left[I_{R}^{i}(x, y) \vee I_{A}^{i}(y)\right]$ and $F_{\bar{R}(A)}^{i}(x)=\wedge_{y \in V}\left[F_{R}^{i}(x, y) \vee F_{A}^{i}(y)\right]$. Based on the above statements, the pair $(\underline{R}(A), \bar{R}(A))$ is named a SVNRM over two universes.

\subsection{PRSVNMs over Two Universes}

Considering the various advantages of PRSs, we then extend the presented SVNRMs over two universes to the context of PRSs. In what follows, we first investigate rough single-valued neutrosophic multisets on a probabilistic approximation space over two universes.

Definition 9. Suppose that $U, V$ are two universes of discourse, $R \subseteq U \times V$ is a binary relation on two universes, and $P$ is a probabilistic measure based on the $\sigma$ algebra. Then $(U, V, R, P)$ is named a probabilistic approximation space over two universes.

Next, we develop the approach to obtain the conditional probability $P(A \mid R(x))$ of an event expressed by SVNMs given the description $R(x) \in 2^{V}$. 
Definition 10. Suppose that $(U, V, R, P)$ is a probabilistic approximation space. For any $A \in \operatorname{SVNM}(V)$, $x \in U, y \in V$, the conditional probability $P(A \mid R(x))$ is given by

$$
P(A \mid R(x))=\frac{\sum_{y \in R(x)} A(y)}{|R(x)|} .
$$

By virtue of the proposed conditional probability $P(A \mid R(x))$, the definition of PRSVNMs over two universes is put forward as follows.

Definition 11. Suppose that $(U, V, R, P)$ is a probabilistic approximation space over two universes. For any $0 \leq \beta \leq \alpha \leq 1, A \in \operatorname{SVNM}(V), x \in U, y \in V$, the lower and upper approximations of $A$ with respect to $(U, V, R, P)$ are given by

$$
\begin{aligned}
& \underline{\operatorname{SVNM}}_{P}^{\alpha}(A)=\{P(A \mid R(x)) \geq \alpha \mid x \in U, y \in V\}=\left\{\frac{\sum_{y \in R(x)} A(y)}{|R(x)|} \geq \alpha \mid x \in U, y \in V\right\}, \\
& \overline{\operatorname{SVNM}}_{P}^{\beta}(A)=\{P(A \mid R(x))>\beta \mid x \in U, y \in V\}=\left\{\frac{\sum_{y \in R(x)} A(y)}{|R(x)|}>\beta \mid x \in U, y \in V\right\},
\end{aligned}
$$

where the pair $\left(\underline{S V N M}_{P}^{\alpha}(A), \overline{S V N M}_{P}^{\beta}(A)\right)$ is named a PRSVNM over two universes. Moreover, by virtue of the above approximations, the positive region, negative region and boundary region of $A$ are further given by

$$
\begin{aligned}
& \operatorname{POS}_{S V N M}(A, \alpha, \beta)=\underline{\operatorname{SVNM}}_{P}^{\alpha}(A)=\left\{\frac{\sum_{y \in R(x)} A(y)}{|R(x)|} \geq \alpha \mid x \in U, y \in V\right\}, \\
& \operatorname{NEG}_{S V N M}(A, \alpha, \beta)=U-\overline{\operatorname{SVNM}}_{P}^{\beta}(A)=\left\{\frac{\sum_{y \in R(x)} A(y)}{|R(x)|}<\beta \mid x \in U, y \in V\right\}, \\
& \operatorname{BND}_{S V N M}(A, \alpha, \beta)=\overline{\operatorname{SVNM}}_{P}^{\beta}(A)-\underline{\operatorname{SVNM}}_{P}^{\alpha}(A)=\left\{\beta<\frac{\sum_{y \in R(x)} A(y)}{|R(x)|}<\alpha \mid x \in U, y \in V\right\} .
\end{aligned}
$$

\subsection{SVNPRMs over Two Universes}

In the previous descriptions, we explore rough single-valued neutrosophic multisets on a probabilistic approximation space over two universes. However, the probabilistic approximation space over two universes $(U, V, R, P)$ could only express the crisp relation of the elements from the universe $U$ to the universe $V$. Since there exist lots of relations based on SVNMs, it is necessary to study SVNPRMs over two universes. In what follows, we extend the general probabilistic approximation space over two universes to the probabilistic approximation space over two universes on SVNMs,

Definition 12. Suppose that $U, V$ are two universes of discourse, $R \in S V N M(U \times V)$ is a relation on SVNMs, and $P$ is a probabilistic measure based on the $\sigma$ algebra. Then $(U, V, R, P)$ is named a probabilistic approximation space over two universes based on SVNMs.

Definition 13. Suppose that $(U, V, R, P)$ is a probabilistic approximation space over two universes based on SVNMs. For any $A \in \operatorname{SVNM}(V), x \in U, y \in V$, the conditional probability $P(A \mid R(x, y))$ is given by

$$
P(A \mid R(x, y))=\frac{\sum_{y \in V} A(y) R(x, y)}{\sum_{y \in V} R(x, y)} .
$$

By virtue of the proposed conditional probability $P(A \mid R(x, y))$, the definition of SVNPRMs over two universes is put forward as follows. 
Definition 14. Suppose that $(U, V, R, P)$ is a probabilistic approximation space over two universes based on SVNMs. For any $0 \leq \beta \leq \alpha \leq 1, A \in \operatorname{SVNM}(V), x \in U, y \in V$, the lower and upper approximations of $A$ with respect to $(U, V, R, P)$ are given by

$$
\begin{aligned}
& \underline{\operatorname{SVNMR}}_{P}^{\alpha}(A)=\{P(A \mid R(x, y)) \geq \alpha \mid x \in U, y \in V\}=\left\{\frac{\sum_{y \in V} A(y) R(x, y)}{\sum_{y \in V} R(x, y)} \geq \alpha \mid x \in U, y \in V\right\}, \\
& \overline{\operatorname{SVNMR}}_{P}^{\beta}(A)=\{P(A \mid R(x, y))>\beta \mid x \in U, y \in V\}=\left\{\frac{\sum_{y \in V} A(y) R(x, y)}{\sum_{y \in V} R(x, y)}>\beta \mid x \in U, y \in V\right\},
\end{aligned}
$$

where the pair $\left(\underline{\operatorname{SVNMR}}_{p}^{\alpha}(A), \overline{\operatorname{SVNMR}}_{p}^{\beta}(A)\right)$ is named a SVNPRM over two universes. Moreover, by virtue of the above approximations, the positive region, negative region and boundary region of $A$ are further given by

$$
\begin{aligned}
& \operatorname{POS}_{\operatorname{SVNMR}}(A, \alpha, \beta)=\underline{\operatorname{SVNMR}}_{P}^{\alpha}(A)=\left\{\frac{\sum_{y \in V} A(y) R(x, y)}{\sum_{y \in V} R(x, y)} \geq \alpha \mid x \in U, y \in V\right\}, \\
& \operatorname{NEG}_{S V N M R}(A, \alpha, \beta)=U-\overline{\operatorname{SVNMR}}_{P}^{\beta}(A)=\left\{\frac{\sum_{y \in V} A(y) R(x, y)}{\sum_{y \in V} R(x, y)} \leq \beta \mid x \in U, y \in V\right\}, \\
& \operatorname{BND}_{S V N M R}(A, \alpha, \beta)=\overline{\operatorname{SVNMR}}_{P}^{\beta}(A)-\underline{\operatorname{SVNMR}}_{P}^{\alpha}(A)=\left\{\beta<\frac{\sum_{y \in V} A(y) R(x, y)}{\sum_{y \in V} R(x, y)}<\alpha \mid x \in U, y \in V\right\} .
\end{aligned}
$$

It is noted that the parameters $\alpha$ and $\beta$ in the above definitions can be determined in advance by experts based on their experience and knowledge in realistic decision making situations.

According to the above definitions, a simple example is offered as follows.

Example 1. Suppose that $U=\left\{x_{1} \cdot x_{2}, x_{3}\right\}$ and $V=\left\{x_{1} \cdot x_{2}, x_{3}\right\}$ are two universes, $R \in S V N M(U \times V)$ is a relation on $S V N M$ s based on two related universes, where

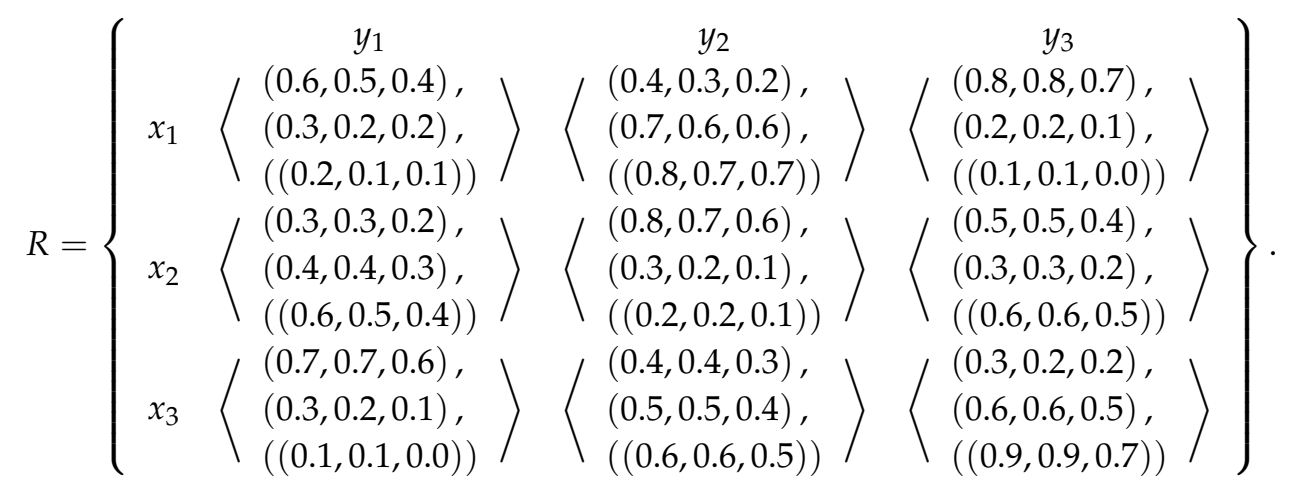

A SVNM A in the universe $V$ is provided below.

$$
A=\left\{\left\langle y_{1},(0.5),(0.5),(0.2)\right\rangle,\left\langle y_{2},(0.8),(0.3),(0.1)\right\rangle,\left\langle y_{3},(0.3),(0.6),(0.7)\right\rangle\right\} .
$$

By virtue of Definition 13, we obtain

$$
\begin{aligned}
& P\left(A \mid R\left(x_{1}, y\right)\right)=\frac{\sum_{y \in V} A(y) R\left(x_{1}, y\right)}{\sum_{y \in V} R\left(x_{1}, y\right)}=\langle(0.67,0.61,0.55),(0.32,0.28,0.27),(0.20,0.14,0.14)\rangle, \\
& P\left(A \mid R\left(x_{2}, y\right)\right)=\frac{\sum_{y \in V} A(y) R\left(x_{2}, y\right)}{\sum_{y \in V} R\left(x_{2}, y\right)}=\langle(0.80,0.76,0.73),(0.23,0.20,0.16),(0.10,0.09,0.07)\rangle, \\
& P\left(A \mid R\left(x_{3}, y\right)\right)=\frac{\sum_{y \in V} A(y) R\left(x_{3}, y\right)}{\sum_{y \in V} R\left(x_{3}, y\right)}=\langle(0.69,0.68,0.64),(0.29,0.29,0.24),(0.13,0.13,0.10)\rangle .
\end{aligned}
$$


If we assume $\alpha=\langle(0.76,0.74,0.72),(0.28,0.27,0.26),(0.13,0.12,0.12)\rangle$ and $\beta=$ $\langle(0.68,0.65,0.6),(0.32,0.31,0.26),(0.18,0.14,0.12)\rangle$, we obtain $\underline{\operatorname{SVNMR}}_{P}^{\alpha}(A)=\left\{x_{2}\right\}$ and $\overline{\operatorname{SVNMR}}_{p}^{\beta}(A)=\left\{x_{2}, x_{3}\right\}$. Then it is not difficult to obtain $\operatorname{POS}_{\operatorname{SVNMR}}(A, \alpha, \beta)=\left\{x_{2}\right\}$, $\operatorname{NEG}_{S V N M R}(A, \alpha, \beta)=\left\{x_{1}\right\}$ and $B N D_{S V N M R}(A, \alpha, \beta)=\left\{x_{3}\right\}$.

In what follows, we show some common properties that are owned by the presented SVNPRMs over two universes.

Proposition 1. Suppose that $(U, V, R, P)$ is a probabilistic approximation space over two universes based on SVNMs. For any $0 \leq \beta \leq \alpha \leq 1, A \in S V N M(V), x \in U, y \in V$, we have the following propositions:

1. $A \subseteq B \Rightarrow \underline{\operatorname{SVNMR}}_{P}^{\alpha}(A) \subseteq \underline{\operatorname{SVNMR}}_{P}^{\alpha}(B), \overline{\operatorname{SVNMR}}_{P}^{\beta}(A) \subseteq \overline{\operatorname{SVNMR}}_{P}^{\beta}(B) ;$

2. $\underline{\operatorname{SVNMR}}_{p}^{\alpha}(\varnothing)=\varnothing, \overline{\operatorname{SVNMR}}_{p}^{\beta}(V)=U$;

3. $\underline{\operatorname{SVNMR}}_{P}^{\alpha}(A) \subseteq \overline{\operatorname{SVNMR}}_{P}^{\beta}(A)$;

4. $\underline{\operatorname{SVNMR}}_{p}^{\alpha}(A \cap B) \subseteq \underline{\operatorname{SVNMR}}_{P}^{\alpha}(A) \cap \underline{\operatorname{SVNMR}}_{P}^{\alpha}(B), \overline{\operatorname{SVNMR}}_{p}^{\beta}(A \cup B) \supseteq \overline{\operatorname{SVNMR}}_{P}^{\beta}(A) \cup$ $\overline{\operatorname{SVNMR}}_{p}^{\beta}(B)$;

5. $\alpha_{1} \leq \alpha_{2} \Rightarrow \underline{\operatorname{SVNMR}}_{P}^{\alpha_{2}}(A) \subseteq \underline{\operatorname{SVNMR}}_{P}^{\alpha_{1}}(A), \beta_{1} \leq \beta_{2} \Rightarrow \overline{\operatorname{SVNMR}}_{P}^{\beta_{2}}(A) \subseteq \overline{\operatorname{SVNMR}}_{P}^{\beta_{1}}(A)$.

The detailed proofs of Proposition 1 are included in the Appendix A at the end of the paper.

\section{Medical Diagnosis Based on SVNPRMs over Two Universes}

\subsection{Medical Diagnosis Model}

In the following, we explore a reasonable and effective medical diagnosis approach by means of SVNPRMs over two universes. As pointed out in the earlier statements, SVNPRMs over two universes take advantage of SVNMs and PRSs at the same time. For one thing, SVNMs are able to provide medical experts with a more powerful tool to describe a complicated medical diagnosis knowledge base, i.e., the SVNMs information could not only handle the uncertain situation well, but also record the diagnostic information at different time intervals reasonably. For another, PRSs-based three-way decisions could further overcome the drawbacks of classical rough sets, and provide a robust decision result by considering the decision risk, hence PRSs-based three-way decisions act as an effectual way to analyze the above SVNMs information.

In the medical diagnosis procedures, suppose that $U=\left\{x_{1}, x_{2}, \ldots, x_{m}\right\}$ is a diagnosis set, and $V=\left\{y_{1}, y_{2}, \ldots, y_{n}\right\}$ is a symptom set. Then based on the universe $U$ and the universe $V$, medical experts are likely to construct the relationship between the diagnosis set and the symptom set by means of the SVNMs information, which is represented by $R \in S V N M(U \times V)$. Moreover, suppose that $P$ is a probabilistic measure based on the $\sigma$ algebra. Hence we establish a medical diagnosis probabilistic approximation space over two universes based on SVNMs $(U, V, R, P)$.

For a given patient, the symptoms of the patient are expressed by a SVNM $A$ in the universe $V$. Next suppose that $\alpha$ and $\beta$ are the thresholds provided in advance by medical experts according to their experience and knowledge in realistic medical diagnosis situations. In light of the above statements, it is not difficult to calculate the two approximations of $A$ in terms of $(U, V, R, P)$, which are denoted by $\underline{S V N M R}_{P}^{\alpha}(A)$ and $\overline{S V N M R}_{P}^{\beta}(A)$. Further, we obtain the positive region, negative region and boundary region of $A$ according to Definition 14, which are expressed as $\operatorname{POS}_{S V N M R}(A, \alpha, \beta)$, $N E G_{S V N M R}(A, \alpha, \beta)$ and $B N D_{S V N M R}(A, \alpha, \beta)$. Lastly, the following medical diagnosis rule could be concluded by virtue of the three-way decisions theory originated by Yao $[49,50]$.

(P) If $x_{i} \in \operatorname{POS}_{\operatorname{SVNMR}}(A, \alpha, \beta), i=1,2, \ldots, m$, then $x_{i}$ is the determined diagnostic conclusion;

(N) If $x_{i} \in N E G_{S V N M R}(A, \alpha, \beta), i=1,2, \ldots, m$, then $x_{i}$ is the excluded diagnostic conclusion; 
(B) If $x_{i} \in B N D_{S V N M R}(A, \alpha, \beta), i=1,2, \ldots, m$, then medical experts are unable to confirm whether $x_{i}$ is the determined or excluded diagnostic conclusion, they need more additional medical examinations to confirm the final diagnostic conclusion

\subsection{Algorithm for Medical Diagnosis Model}

To summarize, we conclude the medical diagnosis approach for a given patient based on SVNPRMs over two universes (Algorithm 1).

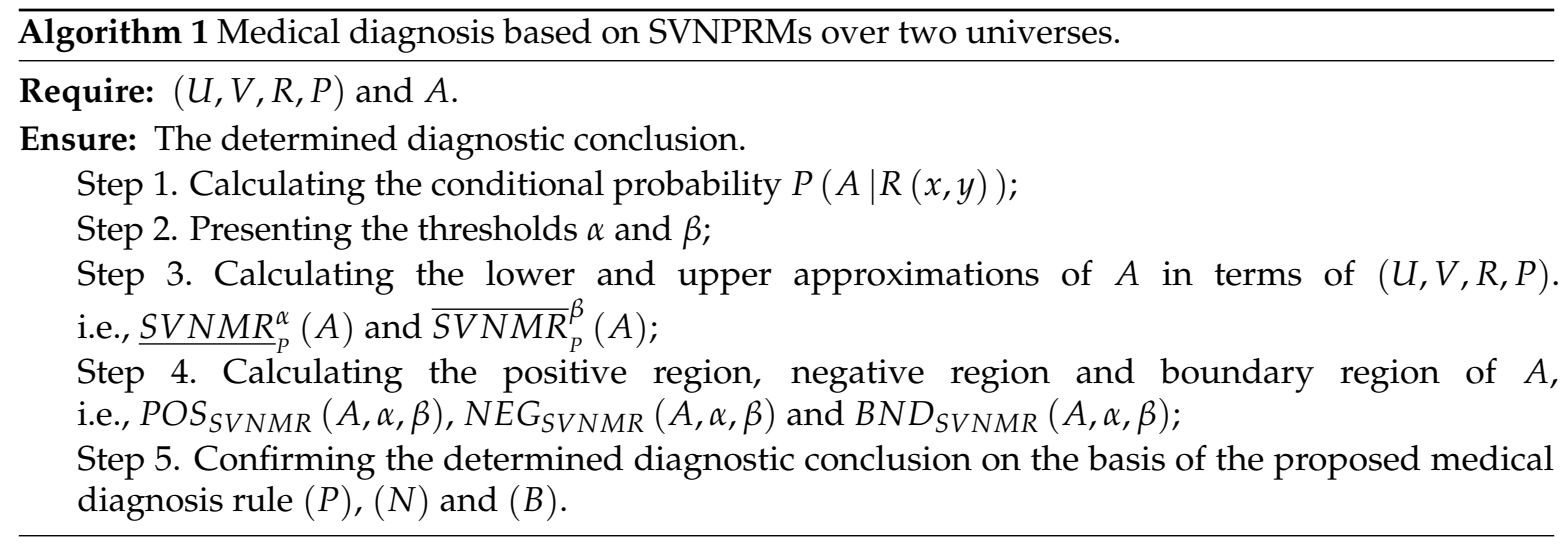

\subsection{An Illustrative Example}

In the following, a case study within the context of medical diagnosis is illustrated as the demonstration of the presented medical diagnosis approach based on SVNPRMs over two universes. The content of the illustrative example (adapted from [26]) is shown as follows.

Let $U=\left\{x_{1}, x_{2}, x_{3}, x_{4}\right\}$ be a diagnosis set (where $x_{i}(i=1,2,3,4)$ denotes "viral fever", "tuberculosis", "typhoid", and "throat disease", respectively), and $V=\left\{y_{1}, y_{2}, y_{3}, y_{4}, y_{5}\right\}$ be a symptom set (where $y_{i}(i=1,2,3,4,5)$ denotes "temperature", "cough", "throat pain", "headache", and "body pain", respectively). Let $R \in S V N M(U \times V)$ be a relation on SVNMs based on two related universes, which is recorded in the Table 1.

Table 1. Relationship between the considered diseases and symptoms represented by SVNMs.

\begin{tabular}{cccccc}
\hline $\boldsymbol{R}$ & $y_{\mathbf{1}}$ & $y_{\mathbf{2}}$ & $y_{3}$ & $y_{4}$ & $y_{5}$ \\
\hline$x_{1}$ & $\langle(0.8),(0.1),(0.1)\rangle$ & $\langle(0.2),(0.7),(0.1)\rangle$ & $\langle(0.3),(0.5),(0.2)\rangle$ & $\langle(0.5),(0.3),(0.2)\rangle$ & $\langle(0.5),(0.4),(0.1)\rangle$ \\
$x_{2}$ & $\langle(0.2),(0.7),(0.1)\rangle$ & $\langle(0.9),(0.0),(0.1)\rangle$ & $\langle(0.7),(0.2),(0.1)\rangle$ & $\langle(0.6),(0.3),(0.1)\rangle$ & $\langle(0.7),(0.2),(0.1)\rangle$ \\
$x_{3}$ & $\langle(0.5),(0.3),(0.2)\rangle$ & $\langle(0.3),(0.5),(0.2)\rangle$ & $\langle(0.2),(0.7),(0.1)\rangle$ & $\langle(0.2),(0.6),(0.2)\rangle$ & $\langle(0.4),(0.4),(0.2)\rangle$ \\
$x_{4}$ & $\langle(0.1),(0.7),(0.2)\rangle$ & $\langle(0.3),(0.6),(0.1)\rangle$ & $\langle(0.8),(0.1),(0.1)\rangle$ & $\langle(0.1),(0.8),(0.1)\rangle$ & $\langle(0.1),(0.8),(0.1)\rangle$ \\
\hline
\end{tabular}

Suppose that the symptoms of the patient is denoted by a SVNM $A$ in the universe $V$ below.

$$
A=\left\{\begin{array}{l}
\left\langle\begin{array}{l}
y_{1},(0.8,0.6,0.5), \\
(0.3,0.2,0.1), \\
(0.4,0.2,0.1)
\end{array}\right\rangle,\left\langle\begin{array}{l}
y_{2},(0.5,0.4,0.3), \\
(0.4,0.4,0.3), \\
(0.6,0.3,0.4)
\end{array}\right\rangle,\left\langle\begin{array}{l}
y_{3},(0.2,0.1,0.0), \\
(0.3,0.2,0.2), \\
(0.8,0.7,0.7)
\end{array}\right\rangle \\
\left\langle\begin{array}{l}
y_{4},(0.7,0.6,0.5), \\
(0.3,0.2,0.1), \\
(0.4,0.3,0.2)
\end{array}\right\rangle,\left\langle\begin{array}{l}
y_{5},(0.4,0.3,0.2) \\
(0.6,0.5,0.5), \\
(0.6,0.4,0.4)
\end{array}\right\rangle
\end{array}\right\} .
$$


According to the steps of the proposed medical diagnosis approach (Algorithm 1), we first calculate the conditional probability as follows.

$$
\begin{aligned}
& P\left(A \mid R\left(x_{1}, y\right)\right)=\frac{\sum_{y \in V} A(y) R\left(x_{1}, y\right)}{\sum_{y \in V} R\left(x_{1}, y\right)}=\langle(0.87,0.74,0.64),(0.07,0.04,0.02),(0.08,0.02,0.01)\rangle, \\
& P\left(A \mid R\left(x_{2}, y\right)\right)=\frac{\sum_{y \in V} A(y) R\left(x_{2}, y\right)}{\sum_{y \in V} R\left(x_{2}, y\right)}=\langle(0.84,0.74,0.61),(0.05,0.03,0.02),(0.07,0.01,0.01)\rangle, \\
& P\left(A \mid R\left(x_{3}, y\right)\right)=\frac{\sum_{y \in V} A(y) R\left(x_{3}, y\right)}{\sum_{y \in V} R\left(x_{3}, y\right)}=\langle(0.75,0.62,0.5),(0.13,0.09,0.06),(0.1,0.03,0.02)\rangle, \\
& P\left(A \mid R\left(x_{4}, y\right)\right)=\frac{\sum_{y \in V} A(y) R\left(x_{4}, y\right)}{\sum_{y \in V} R\left(x_{4}, y\right)}=\langle(0.46,0.34,0.22),(0.15,0.1,0.08),(0.08,0.02,0.01)\rangle .
\end{aligned}
$$

Then we present the thresholds $\alpha$ and $\beta$ below.

$$
\begin{aligned}
& \alpha=\langle(0.85,0.74,0.63),(0.06,0.03,0.02),(0.075,0.02,0.01)\rangle, \\
& \beta=\langle(0.5,0.4,0.3),(0.13,0.1,0.07),(0.09,0.02,0.01)\rangle .
\end{aligned}
$$

Next the two approximations of $A$ in terms of $(U, V, R, P)$ could be obtained.

$$
\begin{aligned}
& \underline{\operatorname{SVNMR}}_{P}^{\alpha}(A)=\{P(A \mid R(x, y)) \geq \alpha \mid x \in U, y \in V\}=\left\{x_{1}\right\}, \\
& \overline{\operatorname{SVNMR}}_{P}^{\beta}(A)=\{P(A \mid R(x, y))>\beta \mid x \in U, y \in V\}=\left\{x_{1}, x_{2}, x_{3}\right\} .
\end{aligned}
$$

Based on the above calculated data, we further obtain

$$
\begin{aligned}
& \operatorname{POS}_{\operatorname{SVNMR}}(A, \alpha, \beta)=\underline{\operatorname{SVNMR}}_{P}^{\alpha}(A)=\left\{x_{1}\right\} \\
& \operatorname{NEG}_{S V N M R}(A, \alpha, \beta)=U-\overline{\operatorname{SVNMR}}_{P}^{\beta}(A)=\left\{x_{4}\right\}, \\
& \operatorname{BND}_{\operatorname{SVNMR}}(A, \alpha, \beta)=\overline{\operatorname{SVNMR}}_{P}^{\beta}(A)-\underline{\operatorname{SVNMR}}{ }_{P}^{\alpha}(A)=\left\{x_{2}, x_{3}\right\} .
\end{aligned}
$$

Finally, we could obtain the diagnostic conclusion by means of the proposed medical diagnosis rule $(P),(N)$ and $(B)$.

$(P)$ The patient is suffering from viral fever, medical experts need to pay close attention to the diagnosis;

(N) The same patient shows no signs of having throat disease, which does not need close attention at the current stage;

(B) Medical experts are unable to confirm whether the considered patient is suffering from tuberculosis and typhoid or not due to insufficient diagnostic information, they might organize an expert consultation to confirm the determined diagnostic conclusion at a later stage.

\subsection{Comparative Analysis}

In what follows, in order to show the applicability and validity of the constructed medical diagnosis approach, we compare the approach based on SVNPRMs over two universes with two significant and common approaches (similarity measures and cosine measures) presented in literature $[26,28]$ respectively.

\subsubsection{Comparison with Other Approaches in Literature [26]}

As presented by Ye et al. [26], suppose that any two SVNMs in the universe $U=\left\{x_{1}, x_{2}, \ldots, x_{m}\right\}$ could be expressed by $A=\left\{\left\langle x_{j}, T_{A}^{i}\left(x_{j}\right), I_{A}^{i}\left(x_{j}\right), F_{A}^{i}\left(x_{j}\right)\right\rangle \mid x_{j} \in U, i=1,2, \ldots, q\right\}$ and $B=$ 
$\left\{\left\langle x_{j}, T_{B}^{i}\left(x_{j}\right), I_{B}^{i}\left(x_{j}\right), F_{B}^{i}\left(x_{j}\right)\right\rangle \mid x_{j} \in U, i=1,2, \ldots, q\right\}$, then the generalized distance measure between $A$ and $B$ is defined as

$$
d_{p}(A, B)=\left[\frac{1}{m} \sum_{j=1}^{m} \frac{1}{3 l_{j}} \sum_{i=1}^{l_{j}}\left(\left|T_{A}^{i}\left(x_{j}\right)-T_{B}^{i}\left(x_{j}\right)\right|^{p}+\left|I_{A}^{i}\left(x_{j}\right)-I_{B}^{i}\left(x_{j}\right)\right|^{p}+\left|F_{A}^{i}\left(x_{j}\right)-F_{B}^{i}\left(x_{j}\right)\right|^{p}\right)\right]^{1 / p},
$$

where $l_{j}=L(x: A, B)=\max \{L(x: A), L(x: B)\}, j=1,2, \ldots, m$. Then based on the generalized distance measure between $A$ and $B$, two similarity measures are defined as

$$
\begin{aligned}
& s_{1}(A, B)=1-d_{p}(A, B) \\
& =1-\left[\frac{1}{m} \sum_{j=1}^{m} \frac{1}{3 l_{j}} \sum_{i=1}^{l_{j}}\left(\left|T_{A}^{i}\left(x_{j}\right)-T_{B}^{i}\left(x_{j}\right)\right|^{p}+\left|I_{A}^{i}\left(x_{j}\right)-I_{B}^{i}\left(x_{j}\right)\right|^{p}+\left|F_{A}^{i}\left(x_{j}\right)-F_{B}^{i}\left(x_{j}\right)\right|^{p}\right)\right]^{1 / p}, \\
& s_{2}(A, B)=\frac{1-d_{p}(A, B)}{1+d_{p}(A, B)} \\
& =\frac{1-\left[\frac{1}{m} \sum_{j=1}^{m} \frac{1}{3 l_{j}} \sum_{i=1}^{l_{j}}\left(\left|T_{A}^{i}\left(x_{j}\right)-T_{B}^{i}\left(x_{j}\right)\right|^{p}+\left|I_{A}^{i}\left(x_{j}\right)-I_{B}^{i}\left(x_{j}\right)\right|^{p}+\left|F_{A}^{i}\left(x_{j}\right)-F_{B}^{i}\left(x_{j}\right)\right|^{p}\right)\right]^{1 / p}}{1+\left[\frac{1}{m} \sum_{j=1}^{m} \frac{1}{3 l_{j}} \sum_{i=1}^{l_{j}}\left(\left|T_{A}^{i}\left(x_{j}\right)-T_{B}^{i}\left(x_{j}\right)\right|^{p}+\left|I_{A}^{i}\left(x_{j}\right)-I_{B}^{i}\left(x_{j}\right)\right|^{p}+\left|F_{A}^{i}\left(x_{j}\right)-F_{B}^{i}\left(x_{j}\right)\right|^{p}\right)\right]^{1 / p}}
\end{aligned}
$$

According to the above stated similarity measures, if we take $p=2$, the largest value of similarity measures between the symptoms of the patient and each potential diagnosis could be regarded as the determined diagnostic conclusion. Since the overall ranking result of the similarity measure shows $x_{1} \succ x_{3} \succ x_{2} \succ x_{4}$, the results of two similarity measures indicate the patient is suffering from viral fever, which is identical with the determined diagnostic conclusion obtained from our proposed approach.

\subsubsection{Comparison with Other Approaches in Literature [28]}

In literature [28], the authors mainly proposed a novel decision making method based on cosine measures of SVNMs. We also suppose that any two SVNMs in the universe $U=\left\{x_{1}, x_{2}, \ldots, x_{m}\right\}$ are described as $A=\left\{x_{j},\left(p_{A 1},\left\langle T_{A 1}\left(x_{j}\right), I_{A 1}\left(x_{j}\right), F_{A 1}\left(x_{j}\right)\right\rangle\right),\left(p_{A 2},\left\langle T_{A 2}\left(x_{j}\right), I_{A 2}\left(x_{j}\right), F_{A 2}\left(x_{j}\right)\right\rangle\right), \ldots\right.$, $\left.\left(p_{A 1},\left\langle T_{A i}\left(x_{j}\right), I_{A i}\left(x_{j}\right), F_{A i}\left(x_{j}\right)\right\rangle\right) \mid x_{j} \in U\right\}$ and $B=\left\{x_{j},\left(p_{B 1},\left\langle T_{B 1}\left(x_{j}\right), I_{B 1}\left(x_{j}\right), F_{B 1}\left(x_{j}\right)\right\rangle\right)\right.$, $\left.\left(p_{B 2},\left\langle T_{B 2}\left(x_{j}\right), I_{B 2}\left(x_{j}\right), F_{B 2}\left(x_{j}\right)\right\rangle\right), \ldots,\left(p_{B 1},\left\langle T_{B i}\left(x_{j}\right), I_{B i}\left(x_{j}\right), F_{B i}\left(x_{j}\right)\right\rangle\right) \mid x_{j} \in U\right\}$, where $p$ denotes the repeated times with the same neutrosophic components. Based on that, the cosine measure between two SVNMs $A$ and $B$ is defined as

$$
\rho(A, B)=\frac{1}{m} \sum_{i=1}^{m} \cos \left\{\frac{\pi}{6}\left(\begin{array}{l}
\left|\prod_{k=1}^{i}\left(1-T_{A k}\left(x_{j}\right)\right)^{p_{A k}}-\prod_{k=1}^{i}\left(1-T_{B k}\left(x_{j}\right)\right)^{p_{B k}}\right|+ \\
\left|\prod_{k=1}^{i}\left(I_{A k}\left(x_{j}\right)\right)^{p_{A k}}-\prod_{k=1}^{i}\left(I_{B k}\left(x_{j}\right)\right)^{p_{B k}}\right|+ \\
\left|\prod_{k=1}^{i}\left(F_{A k}\left(x_{j}\right)\right)^{p_{A k}}-\prod_{k=1}^{i}\left(F_{B k}\left(x_{j}\right)\right)^{p_{B k}}\right|
\end{array}\right)\right\},
$$

As described in the case study section in literature [28], one customer intends to purchase a car, let $U=\left\{x_{1}, x_{2}, x_{3}, x_{4}\right\}$ be a car set with four possible alternatives. Then we let $V=\left\{y_{1}, y_{2}, y_{3}, y_{4}\right\}$ be an attribute set (where $y_{i}(i=1,2,3,4)$ denotes "fuel economy", "price", "comfort", and "safety", respectively). Let $R \in S V N M(U \times V)$ be a relation on SVNMs based on two related universes, which is recorded in the Table 2. 
Table 2. Relationship between alternatives and attributes represented by SVNMs for purchasing a car.

\begin{tabular}{ccccc}
\hline $\boldsymbol{R}$ & $y_{1}$ & $y_{2}$ & $y_{3}$ & $y_{4}$ \\
\hline$x_{1}$ & $\langle(0.7,0.5),(0.7,0.3),(0.6,0.2)\rangle$ & $\langle(0.5),(0.4),(0.4)\rangle$ & $\langle(0.8,0.7),(0.7,0.7),(0.6,0.5)\rangle$ & $\langle(0.5,0.1),(0.5,0.2),(0.8,0.7)\rangle$ \\
$x_{2}$ & $\langle(0.9,0.7),(0.7,0.7),(0.5,0.1)\rangle$ & $\langle(0.8),(0.7),(0.6)\rangle$ & $\langle(0.9,0.9),(0.6,0.6),(0.4,0.4)\rangle$ & $\langle(0.5,0.5),(0.2,0.1),(0.9,0.7)\rangle$ \\
$x_{3}$ & $\langle(0.6,0.3),(0.4,0.3),(0.7,0.2)\rangle$ & $\langle(0.2),(0.2),(0.2)\rangle$ & $\langle(0.9,0.6),(0.5,0.5),(0.5,0.2)\rangle$ & $\langle(0.7,0.4),(0.5,0.2),(0.3,0.2)\rangle$ \\
$x_{4}$ & $\langle(0.9,0.8),(0.7,0.6),(0.2,0.1)\rangle$ & $\langle(0.5),(0.3),(0.2)\rangle$ & $\langle(0.5,0.1),(0.7,0.4),(0.5,0.2)\rangle$ & $\langle(0.8,0.8),(0.4,0.4),(0.2,0.2)\rangle$ \\
\hline
\end{tabular}

Suppose that the ideal attribute set is denoted by a SVNM $A$ in the universe $V$ below.

$$
A=\left\{\begin{array}{l}
\left\langle y_{1},(0.98),(0.12),(0.02)\right\rangle,\left\langle y_{2},(0.7),(0.2),(0.2)\right\rangle, \\
\left\langle y_{3},(0.99),(0.16),(0.1)\right\rangle,\left\langle y_{4},(0.82),(0.02),(0.06)\right\rangle
\end{array}\right\} .
$$

Based on the steps of our proposed approach, we first calculate the conditional probability as follows.

$$
\begin{aligned}
& P\left(A \mid R\left(x_{1}, y\right)\right)=\frac{\sum_{y \in V} A(y) R\left(x_{1}, y\right)}{\sum_{y \in V} R\left(x_{1}, y\right)}=\langle(1,0.97),(0.05,0.02),(0.06,0.02)\rangle \\
& P\left(A \mid R\left(x_{2}, y\right)\right)=\frac{\sum_{y \in V} A(y) R\left(x_{2}, y\right)}{\sum_{y \in V} R\left(x_{2}, y\right)}=\langle(1,0.99),(0.02,0.02),(0.04,0.01)\rangle, \\
& P\left(A \mid R\left(x_{3}, y\right)\right)=\frac{\sum_{y \in V} A(y) R\left(x_{3}, y\right)}{\sum_{y \in V} R\left(x_{3}, y\right)}=\langle(1,0.98),(0.03,0.01),(0.03,0)\rangle, \\
& P\left(A \mid R\left(x_{4}, y\right)\right)=\frac{\sum_{y \in V} A(y) R\left(x_{4}, y\right)}{\sum_{y \in V} R\left(x_{4}, y\right)}=\langle(0.99,0.97),(0.04,0.03),(0,0)\rangle .
\end{aligned}
$$

Then we present the thresholds $\alpha$ and $\beta$ below.

$$
\begin{aligned}
& \alpha=\langle(1,0.99),(0.02,0.02),(0.025,0.02)\rangle, \\
& \beta=\langle(0.98,0.97),(0.03,0.02),(0.02,0.01)\rangle .
\end{aligned}
$$

Next we further calculate the two approximations of $A$ in terms of $(U, V, R, P)$ and the three divided regions.

$$
\begin{aligned}
& \underline{\operatorname{SVNMR}}_{P}^{\alpha}(A)=\{P(A \mid R(x, y)) \geq \alpha \mid x \in U, y \in V\}=\left\{x_{3}\right\}, \\
& \overline{\operatorname{SVNMR}}_{P}^{\beta}(A)=\{P(A \mid R(x, y))>\beta \mid x \in U, y \in V\}=\left\{x_{2}, x_{3}, x_{4}\right\}, \\
& \operatorname{POS}_{S V N M R}(A, \alpha, \beta)=\underline{\operatorname{SVNMR}} \alpha(A)=\left\{x_{3}\right\}, \\
& \operatorname{NEG}_{S V N M R}(A, \alpha, \beta)=U-\overline{\operatorname{SVNMR}}_{P}^{\beta}(A)=\left\{x_{1}\right\}, \\
& \operatorname{BND}_{S V N M R}(A, \alpha, \beta)=\overline{\operatorname{SVNMR}}_{P}^{\beta}(A)-\underline{\operatorname{SVNMR}}{ }_{P}^{\alpha}(A)=\left\{x_{2}, x_{4}\right\} .
\end{aligned}
$$

Lastly, we could obtain the decision making recommendation by means of the proposed three-way decisions rule $(P),(N)$ and $(B)$.

$(P)$ The customer is suggested to purchase the third car;

(N) The same customer is not suggested to by the first car at present;

$(B)$ The same customer is not sure whether the second car and the forth car are the ideal selections, he or she might collect some additional information to make a final conclusion at a later stage.

According to the above stated cosine measures of SVNMs, the largest value of cosine measures between the alternatives and attributes for purchasing a car could be regarded as the final decision making conclusion. It is noted that the overall ranking result of the cosine measure shows $x_{3} \succ x_{4} \succ$ 
$x_{1} \succ x_{2}$, which indicates the customer should buy the third car, the decision making result is also the same as the determined decision making conclusion obtained from our proposed approach.

In conclusion, it is noted that in the above two comparative analyses, though the final decision making result by virtue of our proposed method is the same as the approaches of similarity measures and cosine measures, the overall ranking result by using our proposed method is slightly different from the approaches of similarity measures and cosine measures based on SVNMs. To be specific, if we rank alternatives according to the corresponding values of conditional probability in our presented approach, all the results are shown in the following Tables 3 and 4.

Table 3. The ranking orders by utilizing two different methods in the first comparison analysis.

\begin{tabular}{ccc}
\hline Different Methods & Ranking Results of Alternatives & The Best Alternative \\
\hline Method 1 based on similarity measures in [26] & $x_{1} \succ x_{3} \succ x_{2} \succ x_{4}$ & $x_{1}$ \\
The proposed method & $x_{1} \succ x_{2} \succ x_{3} \succ x_{4}$ & $x_{1}$ \\
\hline
\end{tabular}

Table 4. The ranking orders by utilizing two different methods in the second comparison analysis.

\begin{tabular}{ccc}
\hline Different Methods & Ranking Results of Alternatives & The Best Alternative \\
\hline Method 2 based on cosine measures in [28] & $x_{3} \succ x_{4} \succ x_{1} \succ x_{2}$ & $x_{3}$ \\
The proposed method & $x_{3} \succ x_{2} \succ x_{4} \succ x_{1}$ & $x_{3}$ \\
\hline
\end{tabular}

Compared with the approaches of similarity measures and cosine measures based on SVNMs, the approaches of similarity measures and cosine measures lack the ability of processing decision risks and noisy decision making data. In addition, the proposed approach based on SVNPRMs over two universes offers a reasonable and efficient tool for analyzing the SVNMs information, which not only considers the decision risks by introducing a three-way decision tactic, but also enhances the performance of handling various noisy SVNMs data by introducing the thresholds. Moreover, our presented medical diagnosis approach could be seen as another similarity measures approach, i.e., the conditional probability expresses the similarity of the symptoms of the patient $A$ with the relationship between the considered diseases and symptoms, by further adding the thresholds $\alpha$ and $\beta$, the ability of processing risk preferences of medical experts could be improved. Thus, the constructed approach based on SVNPRMs over two universes is able to enhance the reliability and accuracy of medical diagnosis efficiently.

\section{Conclusions}

In this article, we mainly investigate a PRSs-based method to analyze the SVNMs information within the medical diagnosis context. Specifically, after revisiting several fundamental concepts about SVNMs and PRSs, we first put forward the notion of SVNRMs over two universes and PRSVNMs over two universes. Based on that, the notion of SVNPRMs over two universes is further established. Then some common propositions of the presented SVNPRMs over two universes are further explored. Next, based on the proposed SVNPRMs over two universes, we construct a medical diagnosis approach by means of the three-way decisions strategy. At last, an illustrative case analysis along with a comparative study is carried out to reveal the practicability and effectiveness of the constructed medical diagnosis approach. In future work, it is necessary to establish some more PRSs-based theoretical models based on neutrosophic triplet structures and neutrosophic duplet structures, and it is also meaningful to apply other valid decision making tools to handle various complicated decision making situations.

Author Contributions: C.Z. conducted the modelings and wrote the paper. D.L. provided innovation points of the work. S.B. and A.K. Sangaiah offered plenty of advice for enhancing the readability of the work. All authors approved the publication of the work. 
Funding: This work was supported in part by the National Natural Science Foundation of China (Nos. 61672331, 61432011, 61573231 and U1435212), and the Natural Science Foundation of Shanxi Province (Nos. 201601D021076, 2015091001-0102 and 201601D021072).

Acknowledgments: The authors would like to thank anonymous reviewers for their valuable comments and suggestions which have significantly improved the quality and presentation of this paper. This work was supported in part by the National Natural Science Foundation of China (Nos. 61672331, 61432011, 61573231 and U1435212), and the Natural Science Foundation of Shanxi Province (Nos. 201601D021076, 2015091001-0102 and 201601D021072).

Conflicts of Interest: The authors declare no conflict of interest.

\section{Appendix A}

The proofs of Proposition 1 are listed as follows.

\section{Proof.}

1. Since $A \subseteq B$, according to Definition 14, we have

$\underline{\operatorname{SVNMR}}_{P}^{\alpha}(A)=\left\{\frac{\sum_{y \in V} A(y) R(x, y)}{\sum_{y \in V} R(x, y)} \geq \alpha \mid x \in U, y \in V\right\} \subseteq\left\{\frac{\sum_{y \in V} B(y) R(x, y)}{\sum_{y \in V} R(x, y)} \geq \alpha \mid x \in U, y \in V\right\}=$ $\underline{\operatorname{SVNMR}}_{P}^{\alpha}(B)$.

Thus we obtain $A \subseteq B \Rightarrow \underline{\operatorname{SVNMR}}_{P}^{\alpha}(A) \subseteq \underline{\operatorname{SVNMR}}_{P}^{\alpha}(B)$. Similarly, we could also obtain $\overline{\operatorname{SVNMR}}_{p}^{\beta}(A) \subseteq \overline{\operatorname{SVNMR}}_{p}^{\beta}(B)$.

2. $\underline{\operatorname{SVNMR}}_{P}^{\alpha}(\varnothing)=\left\{\frac{\sum_{y \in V} \varnothing R(x, y)}{\sum_{y \in V} R(x, y)} \geq \alpha \mid x \in U, y \in V\right\}=\varnothing$,

$\overline{\operatorname{SVNMR}}_{P}^{\beta}(V)=\left\{\frac{\sum_{y \in V} V R(x, y)}{\sum_{y \in V} R(x, y)}>\beta \mid x \in U, y \in V\right\}=U$.

Hence $\underline{\operatorname{SVNMR}}_{P}^{\alpha}(\varnothing)=\varnothing$ and $\overline{\operatorname{SVNMR}}_{p}^{\beta}(V)=U$ could be obtained.

3. Since $0 \leq \beta \leq \alpha \leq 1$, it is not difficult to obtain

$\underline{\operatorname{SVNMR}}_{P}^{\alpha}(A)=\left\{\frac{\sum_{y \in V} A(y) R(x, y)}{\sum_{y \in V} R(x, y)} \geq \alpha \mid x \in U, y \in V\right\} \subseteq\left\{\frac{\sum_{y \in V} B(y) R(x, y)}{\sum_{y \in V} R(x, y)}>\beta \mid x \in U, y \in V\right\}=$ $\overline{\operatorname{SVNMR}}_{P}^{\beta}(B)$.

Therefore, $\underline{\operatorname{SVNMR}}_{P}^{\alpha}(A) \subseteq \overline{\operatorname{SVNMR}}_{P}^{\beta}(A)$ could be obtained.

4. If $\underline{\operatorname{SVNMR}}_{P}^{\alpha}(A \cap B)$ holds, we have $P((A \cap B) \mid R(x, y)) \geq \alpha$, then it is not difficult to obtain $\alpha \leq \frac{\sum_{y \in V}(A \cap B)(y) R(x, y)}{\sum_{y \in V} R(x, y)} \leq \frac{\sum_{y \in V} A(y) R(x, y)}{\sum_{y \in V} R(x, y)}$ and $\alpha \leq \frac{\sum_{y \in V}(A \cap B)(y) R(x, y)}{\sum_{y \in V} R(x, y)} \leq \frac{\sum_{y \in V} B(y) R(x, y)}{\sum_{y \in V} R(x, y)}$.

Hence we obtain $\underline{\operatorname{SVNMR}}_{P}^{\alpha}(A \cap B) \subseteq \underline{\operatorname{SVNMR}}_{P}^{\alpha}(A) \cap \underline{\operatorname{SVNMR}}_{P}^{\alpha}(B)$. In an identical fashion, $\overline{\operatorname{SVNMR}}_{P}^{\beta}(A \cup B) \supseteq \overline{\operatorname{SVNMR}}_{P}^{\beta}(A) \cup \overline{S V N M R}_{P}^{\beta}(B)$ could also be obtained.

5. Since $\alpha_{1} \leq \alpha_{2}$, we have

$\underline{\operatorname{SVNMR}}_{P}^{\alpha_{2}}(A)=\left\{\frac{\sum_{y \in V} A(y) R(x, y)}{\sum_{y \in V} R(x, y)} \geq \alpha_{2} \mid x \in U, y \in V\right\} \subseteq\left\{\frac{\sum_{y \in V} A(y) R(x, y)}{\sum_{y \in V} R(x, y)} \geq \alpha_{1} \mid x \in U, y \in V\right\}=$ $\underline{\operatorname{SVNMR}}_{P}^{\alpha_{1}}(A)$.

Hence we have $\alpha_{1} \leq \alpha_{2} \Rightarrow \underline{\operatorname{SVNMR}}_{P}^{\alpha_{2}}(A) \subseteq \underline{\operatorname{SVNMR}}_{P}^{\alpha_{1}}(A)$, and $\beta_{1} \leq \beta_{2} \Rightarrow \overline{\operatorname{SVNMR}}_{P}^{\beta_{2}}(A) \subseteq$ $\overline{S V N M R}_{P}^{\beta_{1}}(A)$ could be proved in a similar way.

\section{References}

1. Mak, D.K. A fuzzy probabilistic method for medical diagnosis. J. Med. Syst. 2015, 39, 26. [CrossRef] [PubMed]

2. Le, H.S.; Thong, N.T. Intuitionistic fuzzy recommender systems: An effective tool for medical diagnosis. Knowl. Based Syst. 2015, 74, 133-150. 
3. Choi, H.; Han, K.; Choi, K.; Ahn, N. A fuzzy medical diagnosis based on quantiles of diagnostic measures. J. Intell. Fuzzy Syst. 2016, 31, 3197-3202. [CrossRef]

4. Zadeh, L.A. Fuzzy sets. Inform. Control 1965, 8, 338-353. [CrossRef]

5. Bustince, H.; Barrenechea, E.; Pagola, M.; Fernandez, J.; Xu, Z.; Bedregal, B.; Montero, J.; Hagras, H.; Herrera, F.; De Baets, B. A historical account of types of fuzzy sets and their relationships. IEEE. Trans. Fuzzy Syst. 2016, 24, 179-194. [CrossRef]

6. Atanassov, K.T. Intuitionistic fuzzy sets. Fuzzy Set. Syst. 1986, 20, 87-96. [CrossRef]

7. Smarandache, F. A Unifying Field in Logics. Neutrosophy: Neutrosophic Probability, Set and Logic; American Research Press: Rehoboth, DE, USA, 1998.

8. Smarandache, F. Neutrosophic logic-generalization of the intuitionistic fuzzy logic. In Extractive Metallurgy of Nickel Cobalt and Platinum Group Metals; Elsevier: New York, NY, USA, 2012; Volume 369, pp. 49-53.

9. Rogatko, A.; Smarandache, F.; Sunderraman, R. A neutrosophic description logic. New Math. Natl. Comput. 2012, 4, 273-290.

10. Kavitha, B.; Karthikeyan, S.; Maybell, S. An ensemble design of intrusion detection system for handling uncertainty using neutrosophic logic classifier. Knowl. Based Syst. 2012, 28, 88-96. [CrossRef]

11. Wang, H.B.; Smarandache, F.; Zhang, Y.Q.; Sunderraman, R. Interval Neutrosophic Sets and Logic: Theory and Applications in Computing; Hexis: Phoenix, AZ, USA, 2005; pp. 1-87.

12. Broumi, S.; Smarandache, F.; Talea, M.; Bakali, A. An introduction to bipolar single valued neutrosophic graph theory. Appl. Mech. Mater. 2016, 841, 184-191. [CrossRef]

13. Broumi, S.; Smarandache, F.; Talea, M.; Bakali, A. Single valued neutrosophic graphs: Degree, order and size. In Proceedings of the IEEE International Conference on Fuzzy Systems, Vancouver, BC, Canada, 24-29 July 2016.

14. Zhang, C.; Zhai, Y.H.; Li, D.Y.; Mu, Y. Steam turbine fault diagnosis based on single-valued neutrosophic multigranulation rough sets over two universes. J. Intell. Fuzzy Syst. 2016, 31, 2829-2837. [CrossRef]

15. Chen, J.Q.; Ye, J. Some Single-valued neutrosophic dombi weighted aggregation operators for multiple attribute decision-making. Symmetry 2017, 9, 82. [CrossRef]

16. Thanh, N.D.; Ali, M.; Le, H.S. A novel clustering algorithm in a neutrosophic recommender system for medical diagnosis. Cogn. Comput. 2017, 9, 526-544. [CrossRef]

17. Ali, M.; Smarandache, F. Complex neutrosophic set. Neural Comput. Appl. 2017, 28, 1817-1834. [CrossRef]

18. Li, X.; Zhang, X.H. Single-valued neutrosophic hesitant fuzzy choquet aggregation operators for multi-attribute decision making. Symmetry 2018, 10, 50. [CrossRef]

19. Abdel-Basset, M.; Mohamed, M.; Smarandache, F. An extension of neutrosophic AHP-SWOT analysis for strategic planning and decision-making. Symmetry 2018, 10, 116. [CrossRef]

20. Wang, Y.; Liu, P. Linguistic neutrosophic generalized partitioned bonferroni mean operators and their application to multi-attribute group decision making. Symmetry 2018, 10, 160. [CrossRef]

21. Zhang, X.; Bo, C.; Smarandache, F.; Park, C. New operations of totally dependent-neutrosophic sets and totally dependent-neutrosophic soft sets. Symmetry 2018, 10, 187. [CrossRef]

22. Ye, S.; Ye, J. Dice similarity measure between single valued neutrosophic multisets and its application in medical diagnosis. Neutrosophic Sets Syst. 2014, 6, 48-53.

23. Yager, R.R. On the theory of bags. Int. J. Gen. Syst. 1986, 13, 23-37. [CrossRef]

24. Smarandache, F. n-Valued Refined Neutrosophic Logic and Its Applications in Physics. Prog. Phys. 2013, 4, 143-146.

25. Chatterjee, R.; Majumdar, P.; Samanta, S.K. Single valued neutrosophic multisets. Ann. Fuzzy Math. Inform. 2015, 10, 499-514.

26. Ye, S.; Fu, J.; Ye, J. Medical diagnosis using distance-based similarity measures of single valued neutrosophic multisets. Neutrosophic Sets Syst. 2015, 7, 47-52.

27. Broumi, S.; Deli, I.; Smarandache, F. Relations on neutrosophic multi sets with properties. arXiv 2015, arxiv:1506.04025. [CrossRef]

28. Fan, C.X.; Fan, E.; Ye, J. The cosine measure of single-valued neutrosophic multisets for multiple attribute decision-making. Symmetry 2018, 10, 154. [CrossRef]

29. Zhang, C.; Li, D.Y.; Yan, Y. A dual hesitant fuzzy multigranulation rough set over two-universe model for medical diagnoses. Comput. Math. Method Med. 2015, 2015; doi:10.1155/2015/292710. [CrossRef] [PubMed] 
30. Guo, Z.L.; Liu, Y.L.; Yang, H.L. A novel rough set model in generalized single valued neutrosophic approximation spaces and its application. Symmetry 2017, 9, 119. [CrossRef]

31. Lu, J.; Li, D.Y.; Zhai, Y.H.; Bai, H.-J. Granular structure of type-2 fuzzy rough sets over two universes. Symmetry 2017, 9, 284. [CrossRef]

32. Sun, B.Z.; Ma, W.M.; Zhao, H.Y. A fuzzy rough set approach to emergency material demand prediction over two universes. Appl. Math. Model. 2013, 37, 7062-7070. [CrossRef]

33. Sun, B.Z.; Ma, W.M. Multigranulation rough set theory over two universes. J. Intell. Fuzzy Syst. 2015, $28,1251-1269$.

34. Zhang, C.; Li, D.Y.; Ren, R. Pythagorean fuzzy multigranulation rough set over two universes and its applications in merger and acquisition. Int. J. Intell. Syst. 2016, 31, 921-943. [CrossRef]

35. Sun, B.Z.; Ma, W.M.; Qian, Y.H. Multigranulation fuzzy rough set over two universes and its application to decision making. Knowl. Based Syst. 2017, 123, 61-74. [CrossRef]

36. Zhang, C.; Li, D.Y.; Mu, Y.M.; Song, D. An interval-valued hesitant fuzzy multigranulation rough set over two universes model for steam turbine fault diagnosis. Appl. Math. Model. 2017, 42, 693-704. [CrossRef]

37. Zhang, C.; Li, D.Y.; Sangaiah, A.; Broumi, S. Merger and acquisition target selection based on interval neutrosophic multigranulation rough sets over two universes. Symmetry 2017, 9, 126. [CrossRef]

38. Zhang, C.; Li, D.Y.; Zhai, Y.H.; Yang, Y. Multigranulation rough set model in hesitant fuzzy information systems and its application in person-job fit. Int. J. Mach. Learn. Cyber. 2017. [CrossRef]

39. Zhang, F.W.; Chen, J.H.; Zhu, Y.H.; Li, J.; Li, Q.; Zhuang, Z. A dual hesitant fuzzy rough pattern recognition approach based on deviation theories and its application in urban traffic modes recognition. Symmetry 2017, 9, 262. [CrossRef]

40. Zeljko, S.; Pamucar, D.; Zavadskas, E.K.; Ćirović, G.; Prentkovskis, O. The selection of wagons for the internal transport of a logistics company: A novel approach based on rough BWM and rough SAW methods. Symmetry 2017, 9, 264.

41. Akram, M.; Ali, G.; Alshehri, N.O. A new multi-attribute decision-making method based on m-polar fuzzy soft rough sets. Symmetry 2017, 9, 271. [CrossRef]

42. Zhang, C.; Li, D.Y.; Liang, J.Y. Hesitant fuzzy linguistic rough set over two universes model and its applications. Int. J. Mach. Learn. Cyber. 2018, 9, 577-588. [CrossRef]

43. Wong, S.K.M.; Ziarko, W. Comparison of the probabilistic approximate classification and the fuzzy set model. Fuzzy Sets Syst. 1987, 21, 357-362. [CrossRef]

44. Yao, Y.Y.; Wong, S.K.M. A decision theoretic framework for approximating concepts. Int. J. Man Mach. Stud. 1992, 37, 793-809. [CrossRef]

45. Ziarko, W. Variable precision rough sets model. J. Comput. Syst. Sci. 1993, 46, 39-59. [CrossRef]

46. Slezak, D.; Ziarko, W. The investigation of the bayesian rough set model. Int. J. Approx. Reason. 2005, 40, 81-91. [CrossRef]

47. Greco, S.; Pawlak, Z.; Slowinski, R. Can bayesian confirmation measures be useful for rough set decision rules? Eng. Appl. Artif. Intel. 2004, 17, 345-361. [CrossRef]

48. Yao, Y.Y.; Zhou, B. Two Bayesian approaches to rough sets. Eur. J. Oper. Res. 2016, 251, 904-917. [CrossRef]

49. Yao, Y.Y. Three-way decisions with probabilistic rough sets. Inform. Sci. 2010, 180, 314-353. [CrossRef]

50. Yao, Y.Y. Three-way decisions and cognitive computing. Cogn. Comput. 2016, 8, 543-554. [CrossRef]

51. Yang, H.L.; Liao, X.W.; Wang, S.Y.; Wang, J. Fuzzy probabilistic rough set model on two universes and its applications. Int. J. Approx. Reason. 2013, 54, 1410-1420. [CrossRef]

52. Sun, B.Z.; Ma, W.M.; Chen, X.T. Fuzzy rough set on probabilistic approximation space over two universes and its application to emergency decision-making. Expert Syst. 2015, 32, 507-521. [CrossRef]

53. Sun, B.Z.; Ma, W.M.; Zhao, H.Y. An approach to emergency decision making based on decision-theoretic rough set over two universes. Soft Comput. 2016, 20, 3617-3628. [CrossRef]

54. Sun, B.Z.; Ma, W.M.; Xiao, X. Three-way group decision making based on multigranulation fuzzy decision-theoretic rough set over two universes. Int. J. Approx. Reason. 2017, 81, 87-102. [CrossRef]

(C) 2018 by the authors. Licensee MDPI, Basel, Switzerland. This article is an open access article distributed under the terms and conditions of the Creative Commons Attribution (CC BY) license (http:// creativecommons.org/licenses/by/4.0/). 